\title{
A Topic Revisited: Students in the Republic of the Maldives Writing Contextual Word Problems
}

\author{
Jason Johnson
}

Zayed University, UNITED ARAB EMIRATES

\begin{abstract}
Students dislike for solving word problems is not new for mathematics teachers. Most word problems have no cultural significance or relate to the student. A student dislike for solving word problems could be contributed to the lack of reference to the lived experience of the student (i.e., social class, race, ethnicity, mother language, gender, sexual orientation, and any other demographic characteristics). A study was designed to explore a group of students, on the island of Kuda Hudaa in the Republic of the Maldives, ability to write contextual word problems. Contextual word problems are word problems that relate to a student population in a classroom. The results indicate that all students were able to create contextual word problems for both multiplication and division. Most student written multiplication and division word problems met Marks (1994) three considerations when developing word problems. The intent is to encourage students to write contextual word problems that make learning mathematics more meaningful for students.
\end{abstract}

KEYWORDS

Ethnomathematics, contextual problems, Maldives, word problems, writing
ARTICLE HISTORY

Received 26 February 2017

Revised 10 July 2017

Accepted 16 July 2017

\section{Introduction}

Far too often, students are engaged with word problems for extra credit or as challenge problems. The word problem is very common in the mathematics classroom. Students' dislike for solving word problems is not a new topic for the mathematics teacher. Most word problems have no cultural significance or relate to the student. Could this dislike come from student lack of connection to the word problems? Similarly, students' dislike for solving word problems is not new for researchers; some argue the reason for student difficulty is due to a linguistic deficiency, while others claim a psychological perspective. The linguistic point of view suggests students' difficulty with word problems

CORRESPONDENCE Jason Johnson $\square$ jason.johnson@zu.ac.ae

(c) 2017 J. Johnson.

Open Access terms of the Creative Commons Attribution 4.0 International License apply. The license permits unrestricted use, distribution, and reproduction in any medium, on the condition that users give exact credit to the original author(s) and the source, provide a link to the Creative Commons license, and indicate if they made any changes. (http://creativecommons.org/licenses/by/4.0/) 
originate from student unable to read, comprehend, and determine which information is needed to solve the problem (Basurto, 1999; Heinze, 2005; Rubenstein \& Thompson, 2002; Winograd \& Higgins, 1994). Conversely, the psychological position explains the difficulty of students' inability to transform word problems into mathematical symbolism (Burton, 1991; Mangan, 1989; Hoz \& Harel, 1990; Puchalska \& Semadeni, 1987). In the end, students continue find answering word problems a laborious task.

I argue that a student dislike for solving word problems is contributed to the lack of reference to the lived experience of the student (i.e., social class, race, ethnicity, mother language, gender, sexual orientation, and any other demographic characteristics) (D'Ambrosio, 1985; 2001; 2009). Meaningful word problems are beneficial for student learning mathematics (Martinez, 2001; Amit \& Klass-Tsirulnikov, 2005). Each student's lived experience is rich in mathematical symbolism (Presmeg, 1998). Students find themselves solving word problems that do not relate to their lived experience. One way to address this is to have students write contextual word problems based on their life. This article will address a study that examined word problems created by students and analyzed using Marks (1994) three suggestions when creating word problems to determine if the word problems have educational merit.

\section{Review of the Literture}

Why is the word problem important for students to make sense of mathematics?

Word problems allow students to use problem solving strategies, rather then, memorized techniques to solve routine problems (Xin, 2007). Students are free to think creatively and make conjectures. Students realize that a creative imagination is beneficial when solving word problems (Foley, Parmar, \& Cawley, 2004). Word problems encourage students to think mathematically and develop reasoning skills rather than rely on prescribed formulas (Martinez, 2001). Word problems also provide students with real-word scenarios (Amit \& KlassTsirulnikov, 2005; Darby, 2008). Mathematics teachers are very familiar with the student question, "when am I ever going to use this?" which is easily addressed with a word problem.

\section{Why is writing to learn mathematics important for students?}

Students who write in mathematics have an opportunity to communicate their mathematical thinking to other students and teacher (Miller; 1991,1992; Winograd and Higgins, 1994). Student writing in mathematics decreases math anxiety (Stix, 1994), encourage student ownership for their learning (Powell and Lopez, 1989), help retain information (DiPillo, Sovich, \& Moss; 1997), and increase higher-order cognition skills (LeGere, 1991).

\section{Why is writing word problems important for students?}

Students who write word problems experience confidence and ability to solve future word problems (Winograd and Higgins, 1994). Even the weaker writer experiences enthusiasm for writing his/her own word problems, since the student is the creator of the word problem (Winograd and Higgins, 1994). Students who write word problems tend to be better problem solvers (Rudnitsky, 
Etheredge, Freeman, \& Gilbert, 1995) and are extremely motivated to solve word problems (Brown, 1993).

\section{Purpose}

Many researchers have described the act of writing as a means to explore mathematics (Miller; 1991,1992; Winograd and Higgins, 1994; Brown, 1993) and many have alluded to the importance of the word problem for students to make sense of mathematics (Amit \& Klass-Tsirulnikov, 2005; Darby, 2008, Xin, 2007). The present study combined both perspectives in an effort to show if students could write contextual mathematics word problems and if these word problems have educational merit. The research question was: are students on the island of Kuda Huraa in the Republic of the Maldives able to write contextual mathematics word problems that have educational value?

\section{The Republic of the Maldives}

The Republic of the Maldives is a country that is made of 1190 coral islands and a double chain of 26 atolls, covering 90,000 square kilometers. Not all islands are habitable. The capital and the largest city is Malé. The country's religion is Sunni Muslim and the spoken language is Dhivehi. However, many Maldivians speak English. The estimated population of the Maldives is around 379,000 (2008). Tourism and fishing are major industries. The country's weather consists of two seasons: dry (January to March) and wet (mid-May to November). The average temperature is 86 degrees Fahrenheit year-round regardless of season.

\section{Education in the Maldives}

The teaching of mathematics in the Maldives has many similarities to other countries. Currently there is a National curriculum for grades $\mathrm{K}-12$, although, grades K-10 are compulsory. The school week is from Sunday through Thursday. According to the Ministry of Education in the Maldives (website), teachers prepare lessons plans that may include differentiated learning objectives, formative and summative evaluations, Individual Educational Plans (IEPs), measureable learning objectives, student centered lessons, strategies to achieve varied learning objectives, and integrate technology. Most islands are not equipped to educate all grades. For instance, the neighboring island to Kuda Huraa has one school, which has grades K -8 . Students on this island would need to travel to another island to continue secondary education.

\section{Framework}

A study was designed to explore a group of students', on the island of Kuda Hudaa in the Republic of the Maldives, ability to write contextual mathematics word problems that have pedagogical merit. Students were to write one word problem for multiplication and another for division. To determine if the word problems have pedagogical merit, word problems were analyzed based on Marks (1994) three suggestions when creating world problems. Marks suggestions were originally written for mathematics teachers, as a guide to follow when developing word problems. I argue students enter a classroom with the creativity to write word problems that reflect Marks (1994) three suggestions. Which could support the notion that students' written contextual mathematics word problems could have educational merit. Figure 1, illustrates 
Marks (1994) three suggestions for teachers when creating word problems. When possible, a student should have an experience with realistic mathematics word problem(s).

\section{Methodology}

The site location was on the island of Kuda Hudaa in the Republic of the Maldives (August 2013). The island of Kuda Hudaa offers a program for Maldivians who would consider a career in hospitality. This program provides students an array of classes that relate to hospitality profession; such as wine tasting, recreation (beach sports activities), English, and mathematics. It was made aware that by the completion of the program, all students would be required to take a basic mathematics comprehensive examination. Moreover, student success on the basic mathematics comprehensive examination had been decreasing.

During the time of data collection, students attend mathematics class everyday (five days a week) for two hours (3:00 pm to 5:00pm). The student population was 18 male and 1 female. Since grade 10 is compulsory and some islands offer grades 11 and 12, not all students were at the same level. The classroom consisted of 3 large circular tables, where each table could fit six or seven students. The mathematics content explored was: Integers - addition, subtraction, multiplication, and division; Fractions - addition, subtraction, multiplication, and division; and Percents - write, convert, and interpret.

\section{Analysis of Data}

Since the research contained student written contextual mathematics word problems, it was most appropriate to use qualitative data analysis methods (Patton, 2002).

\section{Analysis for Pedagogical Value}

The analysis of the contextual word problems contained four phases. Phase one, several readings of the word problems were conducted. By doing so, any mathematical discrepancies were identified. Phase two, another set of readings was conducted to code the word problems that met or did not meet Marks (1994) three suggestions when creating word problems. This portion of the analysis of data consisted of three sets of codes for each word problem (or three separate readings). The reason for the three sets of codes was to determine if the word problems were coded the same (Patton, 2002). Phase three, another set of readings was conducted to code the word problems that met or did not meet Marks (1994) three suggestions when creating word problems without identifying the previous codes from phase two. Again, this portion of the analysis of data consisted of three additional sets of codes for each word problem. After additional scrutiny of the codes in phases two and three, introductory codes were identified (Patton, 2002). Phase four, contained the final set of readings to code the word problems that met or did not meet Marks (1994) three suggestions for creating word problems without identifying the introductory codes. After further inspection of the introductory codes and the codes in phase four, final codes were identified that would be used to analyze the contextual word problems based on Marks (1994) three suggestions when creating word problems.

\section{Results and Discussion}


The results and discussion section will be divided into two parts. Part one will provide an analysis of the multiplication pedagogical value of the contextual word problems using Marks (1994) three suggestions when creating word problems. Part two will offer an analysis of the division pedagogical value of the contextual word problems using Marks (1994) three suggestions when creating word problems.

\section{Multiplication Pedagogical Value} questions

Suggestion one: Avoid composing exercises that inspire unanswerable

Based on the analysis of the word problems, $100 \%$ of the multiplication word problems displayed Marks (1994) suggestion one for creating word problems (see figure 1).

Since 1995, Zihaan is a famous merchant in Thoddoo, who involves in watermelon business. This week he sells 50 watermelons each day at $5 \mathrm{R}$ each (sic).

What is the total amount of Rf he earns this week?

Figure 1. Example word problem that conveys Marks (1994) suggestion one.

Figure 1, underscores the notion that Zihaan, the famous merchant in Thoddoo, would like to determine his earnings in the current week. Since Zihaan's weekly earnings are based on the week's sales, his earnings will not be the same from week-to-week. Meaning, Zihaan will be required to calculate his weekly earnings. According to Marks (1994), suggestion one indicates any information that is already known to the person in the word problem should not be asked. Zihaan's earnings from week-to-week are not consistent; he does not know what his earnings will be (this information is not known to him). Every week Zihaan must calculate his earnings.

Suggestion two: When possible, state problems in terms of the future instead of the past.

Based on the analysis of the word problems, $100 \%$ of the multiplication word problems demonstrated Marks (1994) suggestion two when creating word problems (see figure 2).

According to statistics, Addu City has approximately 11,000 population at the end of 2006 and if the population keeps on getting double by every two years. What would be the estimated population by 2025 (sic)?

Figure 2. Example word problem that conveys Marks (1994) suggestion two.

Figure 2, illustrates a word problem that requires an answer for year 2015 based on population data of Addu City (as known as Seenu Atoll and Addu Atoll). Of course the population of Addu City is not 11,000; the students who created this word problem simply estimated the population data (the most accurate population data for Addu City is around 32,000 - year 2014). According to Marks (1994), the learner should be empowered to use mathematics as a tool for determining "what should be done" (p.611). Rather, than use mathematics to determine an outcome that cannot be changed. In other words, what would be 
the need for mathematics after the action has been completed? The mathematics would be late - we cannot go back in time and change the outcome; unless the mathematics could be used to prevent future events (i.e., car accidents, hurricanes, etc.).

Suggestion three: The motivation for solving a problem should go beyond idle curiosity. Some hypothetical person should gain some advantage

Based on the analysis of the word problems, $36 \%$ of the multiplication word problems displayed Marks (1994) suggestion three when creating word problems, while $64 \%$ of the multiplication word problems were unable to exhibit Marks (1994) suggestion three. See figure 3, for an example word problem that met Marks (1994) suggestion three.

If one fishing dhoani can catch 200 fish a day, Ahmed who owns a fishing dhoni. How much revenue he will earn if he sell each fish for $\$ 10$ (sic)?

Figure 3. Example word problem that conveys Marks (1994) suggestion three.

Figure 3 demonstrates a word problem where the learner is to calculate Ahmed's revenue from his fishing business. This word problem requires the learner to calculate beyond the idle curiosity. Meaning, rather than calculate the total number of fish all eight dhoni boats have caught; the learner must calculate the revenue. The learner would need to have knowledge for how to calculate the revenue. According to Marks (1994), suggestion three indicates, word problems should encourage learners to use mathematics to accomplish a larger task. For this word problem, the larger task is the revenue for Ahmed's fishing business. This could lead to learners inquiring about the cost of Ahmed's business or the profit.

$64 \%$ of the multiplication word problems did not demonstrate Marks (1994) suggestion three when creating word problems (see figure 4).

There are 15 fisherman in a dhoani. Each fisherman caught 35 fish. How many fish did they catch all together (sic)?

Figure 4. Example word problem that did not convey Marks (1994) suggestion three.

In Figure 4, the multiplication word problem references fifteen fishermen in a dhoani. It continues with, "each fisherman can catch thirty-five fish, how many fish did the fisherman catch together?" According to Marks (1994), suggestion three requires word problems to move beyond idle curiosity. This particular word problem requires the learner to calculate the total number of fish for the fifteen fishermen. However, the word problem does not go any further. This problem does not move beyond idle curiosity.

In conclusion, all multiplication word problems validated Marks (1994) suggestions one and two when creating word problems. Word problems demonstrated answerable questions and posed questions in the future. Although, many of the word problems did not convey Marks (1994) suggestion three when creating word problems. These word problems did not move beyond idle curiosity; the hypothetical person in the word problem did not gain any new 
information.

\section{Division Pedagogical Value}

After the first read through of the word problems, it was noted that $82 \%$ of the division problems were not mathematically sound, while $18 \%$ used division correctly. The word problems that did not use division correctly were put aside, which only left $18 \%$ for data analysis. During the second read through, it was realized that all the division word problems that did not use division correctly did not include a divisor. Meaning, if a learner was to respond to these word problems, no division could be completed; since the learner would not know how many sets or groups to use. However, after further inspection, the data analysis revealed that many of the division word problems did convey Marks (1994) three suggestions for creating word problems. The data analysis of the pedagogical value of the division word problems will include student written word problems with the understanding that most will not include a divisor. See figure 5 for an example.

Ihthisham and Mohammed are two business partners. They want to buy all the islands in the Maldives. There are 1998 islands totally in the Maldives. If they share the islands with each other, how man islands can each get (sic)?

Figure 5. Example word problem that lacks a divisor.

Figure 5, illustrates a division word problem which lacks a divisor. In the word problem, Ihthisham and Mohammed want to buy all the islands in the Maldives and divide them. Though, there is no indication how to divide them. Share them equally? Does one get more islands than the other? Again, the data analysis of the pedagogical value of the division word problems will include student written word problems with the understanding that most will not include a divisor. questions

Suggestion one: Avoid composing exercises that inspire unanswerable

Based on the analysis of the word problems, $100 \%$ of the division word problems exhibited Marks (1994) suggestion one for creating word problems. See figure 6 for an example word problem.

This division word problem used the national tree of the Maldives - the coconut tree (see figure 6).

In a coconut palm tree there are around 156 coconuts and 4 coconuts out of them are too young to be used. So, if there are 10 people in total, how many coconuts will each get (sic)?

Figure 6. Example word problem that conveys Marks (1994) suggestion one.

According to the students who wrote this problem, the leaves and timber from the coconut tree is currently used in housing construction and building various tools (i.e., kitchen utensils, jewelry, candle holders, etc.). According to Marks (1994), suggestion one indicates that the learner should not answer questions that the hypothetical person in the word problem should already 
know. For instance, in the above word problem, ten people are to divided amongst them. The people in the word problem should not know how many coconuts each should get. Subsequently, each coconut tree does not produce the same amount of coconuts.

Suggestion two: When possible, state problems in terms of the future instead of the past.

Based on the analysis of the word problems, $100 \%$ of the division word problems demonstrated Marks (1994) suggestion two for creating word problems (see figure 7).

Zihaan has to share each month's earning with his 3 children - Rishma, Ifaz, Moosa. This month he earned Rf. 15,000. How much money will each get (sic)?

Figure 7. Example word problem that conveys Marks (1994) suggestion two.

Zihann, in the division word problem above, wants to take his month's earnings and divide his earnings among his three children (see figure 7 ). The Maldivian currency amount of 15,000 is around 975 USD. This amount is critical to the word problem and the Maldives. According to the students who created this word problem, this month's earnings is realistic for many the Maldivians in the current economy. Another critical perspective to this word problem is Zihaan sharing his earnings with his children. The students talked of family members who work solely for the family and not for individual gain. The money is then divided appropriately among all family members. According to Marks (1994), suggestion two requires word problems to be written in terms of the present or future and not the past. Given the above analysis of the word problem, the students have managed to capture the current economics status of the Maldives.

Suggestion three: The motivation for solving a problem should go beyond idle curiosity. Some hypothetical person should gain some advantage

Based on the analysis of the word problems, $55 \%$ of the division word problems displayed Marks (1994) suggestion three for creating word problems, while $45 \%$ of the division word problems were unable to exhibit Marks (1994) suggestion three. One example of a division word problem that met Marks (1994) suggestion three (see figure 8).

Thoddoo is the most famous and specialize in watermelon farming island in Maldives. And if for a day they can get 500 watermelons for selling and there are only two customers for them. If they sell them equally among two customers, how much does each customer get (sic)?

Figure 8. Example word problem that conveys Marks (1994) suggestion three.

Thoddoo is an island in the Maldives that produces the most watermelons in the country (see figure 8). The students who are from Thoddoo, agreed that packaging five hundred watermelons for sell for two transactions, is realistic; typically other islands would purchase similar quantities. This word problem does provide a divisor - each customer is to receive equal shares of watermelons. Marks (1994) suggestion three, recommends word problems should move beyond idle curiosity. The above word problem implies when selling five hundred 
watermelons, the funds are paid to the island of Thoddoo. Though there is no indication how the money will be divided or used on the island. Since the word problem provided a context about the island of Thoddoo and the sale of the watermelons, it was noted that the word problem does go beyond idle curiosity.

Conversely, $45 \%$ of the division word problems did not demonstrate Marks (1994) suggestion three when creating word problems (see figure 9). The division word problem above (see figure 9), there are twelve fishermen on an island and only 4 dhoani. How many fishermen would fit in each dhoani? According to Marks (1994), suggestion three requires word problems to move beyond idle curiosity. This particular word problem does not indicate which island or any information regarding the fishermen. Though a learner would be able to calculate the number of fishermen for each dhoani.

In an island there are 12 fisherman. But there are only 4 dhoani. So, how many fisherman can get into each dhoani (sic)?

Figure 9. Example word problem that does not convey Marks (1994) suggestion three.

Lastly, all division word problems conveyed Marks (1994) suggestions one and two when creating word problems. Word problems demonstrated answerable questions and posed questions in the future or present. Although, many of the word problems did not display Marks (1994) suggestion three when creating word problems. These word problems did not move beyond idle curiosity; the hypothetical person in the word problem did not gain any new information.

\section{Conclusion}

The current research study came as a result of many conversations with practicing teachers who have described not having enough time to create word problems that reflect the identity of their students. This supports the notion presented by Presmeg (1998) that the "dominant culture of the teacher becomes the only culture valued in the classroom" ( $p$ 319). The intend of this research study is twofold: 1) explore the idea to allow students opportunities to write contextual mathematics word problems, 2) determine if the student written contextual word problems have educational merit. Students were required to write two contextual word problems: multiplication and division. All contextual word problems (multiplication and division) were able to capture Marks (1994) suggestions one and two for creating word problems. The notion that students were able to write word problems that reflect answerable questions and stated in the future provides evidence that such contextual word problems have educational merit; with the exception of Marks (1994) suggestion three. Some word problems did not move beyond the idle curiosity. One reason could be students' lack of experience with word problems that require a greater understanding of a problem, rather, then a simple calculation. The intent is to encourage students to write contextual word problems that make learning mathematics more meaningful for students.

\begin{tabular}{|l|l}
\hline Suggestion one & Avoid composing exercises that inspire unanswerable
\end{tabular} 


\begin{tabular}{|l|l|}
\hline & questions \\
\hline Suggestion two & $\begin{array}{l}\text { When possible, state problems in terms of the future } \\
\text { instead of the past }\end{array}$ \\
\hline Suggestion three & $\begin{array}{l}\text { The motivation for solving a problem should go beyond } \\
\text { idle curiosity. Some hypothetical person should gain } \\
\text { some advantage }\end{array}$ \\
\hline
\end{tabular}

Table: Marks (1994) three suggestions when creating word problems

\section{Disclosure statement}

No potential conflict of interest was reported by the authors.

\section{Notes on contributors}

Jason Johnson - Zayed University, United Arab Emirates

\section{References}

Amit, M. \& Klass-Tsirulnikov, B. (2005). Paving a way to algebraic word problems using a nonalgebra route. Mathematics Teaching in the Middle School, 10, $271-276$.

Basurto, I. (1999). Conditions of reading comprehension which facilitate word problems for second language learners. Reading Improvement, 36(3), 143 - 148.

Brown, N. M. (1993). Writing mathematics. Arithmetic Teacher, 41(1), 20 - 21.

Burton, M. B. (1991). Grammatical translation-inhibitors in two classic word problem sentences. For the Learning of Mathematics, 11(1), $43-46$.

D'Ambrosio, U. (1985). Ethnomathematics and its place in the history and pedagogy of mathematics. For the Learning of Mathematics, 5, 44-8.

D'Ambrosio, U. (2001). What is Ethnomathematics and how can it help children in schools? Teaching Children Mathematics, 7(6), 308-310.

D'Ambrosio, U. (2009). The program Ethnomathematics: A theoretical basis of the dynamics of intra cultural encounters. Journal of Mathematics and Culture. 1, (1), $1-7$.

Darby, L. (2008). Marking mathematics and science relevant through story. Australian Mathematics Teacher, 64(1), $6-11$.

DiPillo, M. L., Sovich, R., \& Moss, B. (1997). Exploring middle graders' mathematical thinking through journals. Mathematics Teaching in the Middle School, 2 (5), $308-314$.

Edie, R. (2009). Making sense of word problems (Master's Thesis). University of Nebraska - Lincoln. Retrieved from: http://digitalcommons.unl.edu/mathmidactionresearch/42/

Foley, T. E., Parmar, R. S., \& Cawley, J. F. (2004). Explanding the agenda in mathematics problem solving for students with mild disabilities: alternative representations. Learning Disabilities, 13(1), $7-16$.

François, K. (2009). The role of Ethnomathematics within mathematics education. Proceedings of the Sixth Congress of the European Society for Research in Mathematics Education (CERME). January 28 - February 1, 2009, Lyon France. <www.inrp.fr/editions/cerme6>

Heinze, K. (2005). The language of math. Presentation handouts from TESOL conference. Retrieved from http://www.rtmsd.org/cms/lib/PA01000204/Centricity/Domain/594/Language_of_Math.doc. 
Hoz, R. \& Harel, G. (1990). Higher order knowledge involved in the solution of algebra speed word problems. Journal of Structural Learning, 10(4), $305-328$.

Langeness, J. (2011). Methods to improve student ability in solving math word problems (Master's Thesis). Hamline University. Retrieved from: http://www.hamline.edu/WorkArea/DownloadAsset.aspx?id=2147514388.

LeGere, A. (1991). Collaboration and writing in the mathematics classroom. Mathematics Teacher. $84(3), 166-171$.

Marks, D. (1994). A guide to more sensible word problems. The Mathematics Teacher, 87(8), 610 611.

Mangan, C. (1989). Choice of operation in multiplication and division word problems: A developmental study. Journal of Structural Learning, 10, $73-77$.

Martinez, J. G. R. (2001). Thinking and writing mathematically: Achilles and the tortoise as an algebraic word problem. Mathematics Teacher, 94(4), 248-252.

Miller, L. D. (1991). Constructing pedagogical content knowledge from students' writing in secondary mathematics. Mathematics Education Research Journal, 3(1), 30 - 44.

Miller, L. D. (1992). Teacher benefits from using impromptu writing prompts in algebra classes. Journal for Research in Mathematics Education, 23(4), 329 - 340.

Patton, M. Q. (2002). Qualitative research and evaluation methods (3rd Ed.). London: Sage Publications.

Powell, A.B., \& Lopez, J.A. (1989). Writing as a vehicle to learn mathematics: A case study. In P. Connolly \& T. Vilardi (Eds.), Writing to learn mathematics and science (pp. 157-177). New York: Teachers College Press.

Presmeg, N. (1998). Ethnomathematics in teacher education. Journal of Mathematics Teacher Education, 1, $317-339$.

Puchalska, E. \& Semadeni, Z. (1987). Children's reactions to verbal arithmetical problems with missing, surplus or contradictory data. For the Learning of Mathematics, 7(3), 9- 16.

Rubenstein, R. N. \& Thoompson, D. R. (2002). Understanding and supporting children's mathematical vocabulary development. Teaching Children Mathematics, 9(2), $107-112$

Rudnitsky, A., Etheredge, S., Freeman, S. J. M., and Gilbert, T. G. (1995). Learning to solve addition and subtraction word problems througha structure-plus-writing approach. Journal for Research in Mathematics Education, 26(5), 467 - 486.

Stix, A. (1994). Pic-jour math: Pictorial journal writing in mathematics. Arithmetic Teacher, 41(5), $264-269$.

Wedege, T. (2010). Ethnomathematics and mathematical literacy: People knowing mathematics in society. C. Bergsten, E. Jablonka, and T. Wedege (eds). Mathematics and mathe-matics education: Cultural and social dimensions. $31-46$.

Winograd, K., \& Higgins, K. (1994). Reading, writing and talking mathematics: One interdisciplinary possibility. The Reading Teacher, 48, $310-319$.

Xin, Y. P. (2007). Word problem solving tasks in textbooks and their relation to student performance. The Journal of Educational Research, 100(6), 347 - 359. 